\title{
超小晶粒锡掺杂 $\mathrm{CsPbBr}_{3}$ 蓝光量子点的合成 \\ 及其光学性能研究
}

曾凡菊 ${ }^{12)}{ }^{2}$, 谭永前 ${ }^{1)}$, 胡伟 ${ }^{2)} \dagger$, 唐孝生 ${ }^{2) 3)}$, 张小梅 ${ }^{1)}$, 尹海峰 ${ }^{1)}$

1) (凯里学院大数据工程学院, 凯里 556011)

2）（重庆大学光电工程学院, 重庆 400044)

3）（重庆邮电大学光电学院，重庆 400065）

\section{摘 要}

近年来, 铅卤钙铁矿 $\mathrm{CsPbX}_{3}(\mathrm{X}=\mathrm{Cl}, \mathrm{Br}$ 或 I ) 因其发光波段敂调、荧光量 子产率高 (Photoluminescence quantum yield, PLQY) 以及花光半峰宽窄等优点 而被广泛应用于光电器件领域。然而, 与 PLQY 接近于 $100 \%$ 的绿光和红光相比, 蓝光卤素钙铁矿的 PLQY 仍比较低。在此, 采用过饱和结晶的方法在室温下合成 了粒径低于 $4 \mathrm{~nm}$ 的超小晶粒锡 $(\mathrm{Sn})$ 掺杂 $\mathrm{CsPbBr}_{3}$ 量子点, 并对其结构特性和 发光特性进行了研究。结果表明: 随着 $\mathrm{SnBr}_{2}$ 添加量的增加, 量子点晶粒粒径略 微减小, 苂光发射峰发生蓝移, 粒径由添加 $\mathrm{SnBr}_{2}$ 为 $0.03 \mathrm{mmol}$ 时的 $3.33 \mathrm{~nm}$ 减 小到 $2.23 \mathrm{~nm}$ ( $\mathrm{SnBr}_{2}$ 为 $0.06 \mathrm{mmol}$ 时), 对腐的苂光发射峰由 $490 \mathrm{~nm}$ 蓝移至 472 $\mathrm{nm}$ 。当添加 $\mathrm{SnBr}_{2}$ 为 $0.05 \mathrm{mmol}$ 时合成的超尔晶粒锡掺杂 $\mathrm{CsPbr}_{3}$ 量子点显示出 最优的光学性能, 其粒径约为 $2.91 \mathrm{~mm}$, 付应的 XRD 各晶面衍射峰强度最强, 苂 光发射峰位于 $472 \mathrm{~nm}$ 处, PLQY 最高, 达到了 $53.4 \%$, 在空气中存放 15 天后, 其苂光发射峰位置不发生明显改变, 苂光 PLQY 仍保留最初的 $80 \%$, 为 $42.7 \%$ 。 证明适量添加 $\mathrm{SnBr}_{2}$ 对 CsPbBr 3 进行锡掺杂可有效提高超小晶粒量子点的结晶性 能和光学性能。

关键词: 超小晶粒; 锡掺杂 $\mathrm{CsPbBr} 3$ 量子点; 合成; 蓝光; 光学性能; 苂光稳定 PACS: 74. 25. Gz, 78. 20. Ci, 42.25. $-\mathrm{p}, 42.50 . \mathrm{Ct}$

基金: 贵州省科技计划项目 (批准号: ZK [2021]245), 国家自然科学基金(批准号: 61975023, 61875211, 51 602033, 61520106012), 凯里学院博士专项课题(批准号: BS202004, BS201301)、 凯里学院学术新苗培养及创新探索专项课题 (批准号: 黔科合平台人才 [2019]01-4) 和贵州省 教育厅创新群体重大研究项目 (批准号: 黔教合 KY 字 [2018]035) 资助的课题.

$\dagger$ 通讯作者. E-mail: zengfan ju@cqu. edu. cn, weihu@cqu. edu. cn, xstang@cqu. edu. cn 


\section{1 引言}

全无机铅卤钻铁矿 $\mathrm{CsPbX}_{3}(\mathrm{X}=\mathrm{Cl}, \mathrm{Br}, \mathrm{I})$ 因其合成工艺简单、带隙可调、 苂光量子产率（Photoluminescence quantum yield, PLQY) 高以及苂光发射峰窄 等优点在发光二极管、光电响应和微激光等光电领域引起了广泛关注 ${ }^{[1-6]}$ 。荧光 量子产率 (Photoluminescence quantum yield, PLQY) 是荧光材料发射光子数与 吸收光子数的比值, 主要用来定量反应荧光材料的发光能力 ${ }^{[7]}$ 。荻光材料的晶体 结构、表面缺陷或晶格畸变等均会影响苂光材料的 PLQY。与PLQY 接近于 $100 \%$ 的绿光 $\left(\mathrm{CsPbBr}_{3}\right)$ 与红光 $\left(\mathrm{CsPbI}_{3}\right)$ 量子点比较, 作为提高色域关键颜色的蓝光 $\mathrm{CsPbCl}_{3}$ 荧光量子产率较低 $(10 \%)$, 且结构极不稳定, 严重阻碍了钻钛矿发光二 极管在全彩显示中的应用 ${ }^{[8-11]}$ 。铅卤钻钛矿 $\mathrm{CsPbCl}$ 的 PLQY 较低主要是由于 $\mathrm{CsPbCl}_{3}$ 化学键为离子型且具有较大的表直能, 导致 $\mathrm{CsPbCl}_{3}$ 对极性溶剂如水、 乙醇和丙酮敏感, 对其发光性质产表个负面影响 ${ }^{[2]}$ 。因此, 如何提高蓝光铅卤钻 铁矿量子点的发光性能仍存在挑战。

为了提高蓝光铅卤鿭铁矿量子点的发光性能, 报道的方法主要有：减小 $\mathrm{CsPbBr}_{3}$ 量子点粒径, 使量子点发光蓝移, 不足的是, 由于量子局域效应, 其蓝 光很不稳定, 空气中晹发生红移, 变成绿光发射 ${ }^{[12,13]}$ 。其次, 采用卤素离子交换 的方法合成蓝光 $\mathrm{CsPbBr}{ }_{x} \mathrm{Cl}_{1-\mathrm{x}}$ 量子点, 但由于晶格的不匹配导致 $\mathrm{CsPbBr}_{x} \mathrm{Cl}_{1-\mathrm{x}}$ 量 子点晶体结构不稳定 ${ }^{[14]}$ 。最近几年, 报道了引入离子半径小的二价或三价金属离 子 $\mathrm{M}^{2+/ 3+}$ 对铅卤钻钣矿中的铅 $\mathrm{Pb}^{2+}$ 离子进行部分替代, 该方法可有效提高钻钣矿 量子点的激子结合能, 合成具有优异光学性能的蓝光钻钛矿量子点。例如, 对 $\mathrm{CsPbX}_{3}$ 晶体结构进行 $\mathrm{Cu}^{2+} 、 \mathrm{Mn}^{2+} 、 \mathrm{Sn}^{2+} 、 \mathrm{Cd}^{2+} 、 \mathrm{Zn}^{2+}$ 、和 $\mathrm{Al}^{3+}$ 等离子掺杂后, 均 不同程度地降低了铅卤钙钛矿缺陷密度, 钝化了量子点晶界, 提高了量子点的发 
光性能 ${ }^{[15-20]}$ 。其中, Ward van der Stam 等 ${ }^{[17]}$ 采用的金属离子交换法合成了 PLQY 高达 $62 \%$ 的 $\mathrm{Sn}^{2+}$ 离子掺杂 $\mathrm{CsPb}_{1-\mathrm{x}} \mathrm{Sn}_{\mathrm{x}} \mathrm{Br}_{3}$ 蓝光量子点 (479 nm), 证明锡掺杂 $\mathrm{CsPbBr}_{3}$ 量子点可有效提高蓝光量子点的发光性能。不足的是, 该方法需要在高 温加热且氮气保护条件下, 采用热注入法合成未掺杂的 $\mathrm{CsPbBr}_{3}$ 量子点, 而后在 常温下采用离子交换法合成 $\mathrm{CsPb}_{1-\mathrm{x}} \mathrm{Sn}_{\mathbf{x}} \mathrm{Br}_{3}$ 蓝光量子点, 合成工艺复杂且成本高 [21]。最近两年, Xiangtong Zhang 等 ${ }^{[22]}$ 在常温条件合成了超小纳米颗粒 $(\sim 2 \mathrm{~nm})$ $\mathrm{Sb}^{3+}$ 离子掺杂 $\mathrm{CsPbBr}_{3}$ 量子点, 成功降低了量子点表面缺陷能,, 是离了晶格形成 能和苂光性能，为提高量子点发光性能提供了新的思路。

本论文采用过饱和结晶的方法在室温下合成了粒徐超小的锡掺杂 $\mathrm{CsPbr}_{3}$ 量子点 (低于 $4 \mathrm{~nm}$ ), 并对其结构特性和光学性能进行了研究。结果显示: 随着 $\mathrm{SnBr}_{2}$ 添加量的增加, 合成量子点粒径减水, 岫 $3.33 \mathrm{~nm}\left(\mathrm{SnBr}_{2} 0.03 \mathrm{mmol}\right)$ 减小 到 $2.23 \mathrm{~nm}\left(\mathrm{SnBr}_{2} 0.06 \mathrm{mmol}\right)$, 合成量点菼光峰发生明显蓝移, 由 $490 \mathrm{~nm}\left(\mathrm{SnBr}_{2}\right.$ $0.03 \mathrm{mmol})$ 蓝移至 $472 \mathrm{~nm}\left(\mathrm{SnBr}_{2} 0.06 \mathrm{mmol}\right)$ 。当添加 $\mathrm{SnBr}_{2}$ 为 $0.05 \mathrm{mmol}$ 时, 合成的蓝光 $(472 \mathrm{~nm})$ 量子点PLQY 最高, 为 $53.4 \%$, 苂光发光具有良好的稳定 性, 在空气中存放 15 天合, 其荧光发射峰不发生明显改变, 仍位于 $472 \mathrm{~nm}$ 处, 属于蓝光发射, PLQY 仍保持最初的 $80 \%$, 为 $42.7 \%$ 。该方法合成了蓝光 PLQY 高、空气中发光稳定的超小晶粒蓝光量子点, 为合成光学性能优异的蓝光量子点 提供了参

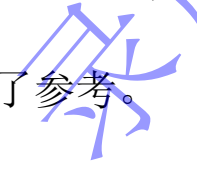

\section{2 实验}

\section{1 实验材料}

溴化铅 $\left(\mathrm{PbBr}_{2}, 99.9 \%\right)$ 和溴化铯 $(\mathrm{CsBr}, 99.9 \%)$ 从西安宝莱特光电科技 有限公司购买。溴化铝 $\left(\mathrm{AlBr}_{3}, 98 \%\right)$ 、油酸 $\left(\mathrm{C}_{18} \mathrm{H}_{34} \mathrm{O}_{2}, \mathrm{OA}, 80 \%\right)$ 、油胺 
$\left(\mathrm{CH}_{3}\left(\mathrm{CH}_{2}\right)_{7} \mathrm{CH}, \mathrm{OAm}, 97 \%\right)$ 和甲苯 $\left(\mathrm{C}_{7} \mathrm{H}_{8} ， 99 \%\right)$ 购于上海阿拉丁生化科技 股份有限公司。二甲基乙酰胺 $\left(\mathrm{C}_{3} \mathrm{H}_{7} \mathrm{NO}, \mathrm{DMF}, 99.8 \%\right)$ 、正己烷 $\left(\mathrm{C}_{6} \mathrm{H}_{14}, 97 \%\right)$ 、 乙酸甲酯（ $\left.\mathrm{C}_{4} \mathrm{H}_{6} \mathrm{O}_{2} ， 95 \%\right)$ 购买于默克 Sigma-Aldrich。

\section{2 超小晶粒锡掺杂 $\mathrm{CsPbBr}_{3}$ 量子点的制备}

称量 $0.4 \mathrm{mmol}(146.8 \mathrm{mg}) \mathrm{PbBr}_{2}$ 和 $0.4 \mathrm{mmol}(85.1 \mathrm{mg}) \mathrm{CsBr}$ 于 $20 \mathrm{~mL}$ 容 积的 $\mathrm{A}$ 试剂瓶, 取 $10 \mathrm{mLDFM}$ 加入试剂瓶, 加入磁力摚拌子, 置于磁力摚拌台 进行搅拌, 直至溶质完全溶解。加入 $1 \mathrm{~mL}$ 油酸和 $0.5 \mathrm{~mL}$ 油胺作为稳定剂, 常温 下搅拌半小时后待用。称量 $1 \mathrm{mmol}\left(278.5 \mathrm{mg}\right.$ ) $\mathrm{SnBr}_{2}$ 于 $5 \mathrm{~mL}$ 容积的 B 试剂瓶, 加入 $1 \mathrm{mLDMF}$ 溶剂及磁力搅拌子 (手套箱), 密封置王磁为摚拌器进行摚拌 (大 气氛围), 直至 $\mathrm{SnBr}_{2}$ 完全溶解。取出 4 个 $20 \mathrm{~mL}$ 容积的试剂瓶, 分别加入 $10 \mathrm{~mL}$ 甲苯及磁力摚拌子, 并置于磁力摚拌台快速摚拌。采用移液枪于 $\mathrm{A}$ 试剂瓶取出 1 $\mathrm{mL} \mathrm{CsPbBr} 3$ 前驱体溶液 $\left(\mathrm{PbBr}_{2}\right.$ 约为 $\left.0.034 \mathrm{mmol}\right)$, 同时从 $\mathrm{B}$ 试剂瓶取出不同量 的 $\mathrm{SnBr}_{2}$ 溶液, 迅速加入甲苯溶剂, 剧烈摚拌 $2 \mathrm{~min}$, 即获得 $\mathrm{Sn}^{2+}$ 离子掺杂 $\mathrm{CsPbBr}_{3}$ 量子点胶体。其中, 添加不同的 $\mathrm{SnBr}_{3}$ 溶液分别为 $30 \mu \mathrm{L}\left(0.03 \mathrm{mmol} \mathrm{SnBr}_{3}\right) 、 40$ $\mu \mathrm{L}\left(0.04 \mathrm{mmol} \mathrm{SnBr}_{3}\right) \quad 50 \mu \mathrm{L}\left(0.05 \mathrm{mmol} \mathrm{SnBr}_{3}\right)$ 或 $60 \mu \mathrm{L}\left(0.06 \mathrm{mmol} \mathrm{SnBr}_{3}\right)$ 。 最后, 采用乙酸甲酯和业己烷（体积比: v:v=3:1）对所合量子点胶体中的剩余反 应物及有机物进行洗涤, 重复 3 次, 将沉淀分散于正己烷, 即可获得 4 种添加不 同 $\mathrm{SnBr}_{2}$ 的锡掺杂 $\mathrm{CsPbr}_{3}$ 量子点。

\section{3 样品表征与光谱测试}

所合成量子点的晶体结构采用 $\mathrm{X}$ 射线衍射仪进行表征（X-ray diffraction， $\mathrm{XRD}, \mathrm{Cu} \mathrm{K \alpha}$ irradiation, $\lambda=0.15406 \mathrm{~nm})$ 。量子点晶体形貌、晶格间距和元素电 子能谱图（Energy Dispersive Spectrometer, EDS）均采用透射电子显微镜 
（Transmission Electron Microscope, TEM）进行表征。吸收光谱、苂光光谱以及 苂光量子产率（Photoluminescence quantum yield, PLQY) 分别采用紫外-可见分 光光度计（UV-2100）和爱丁堡荧光光谱仪（Edinburgh fluorescence spectrometer FS 5 )进行表征。量子点苂光衰减寿命采用苂光寿命测量系统 (QM TM NIR, PTI) 进行表征。所有光学性能测试均在大气氛围进行。

\section{3 结果讨论}

\section{1 结构特性}

图 1 为所合成不同锡掺杂 $\mathrm{CsPbBr}_{3}$ 量子点的 $\mathrm{XRD}$ 图谱。除 $\mathrm{SnBr}_{2}$ 添加量为 $0.06 \mathrm{mmol}$ 时, 锡掺杂 $\mathrm{CsPbBr}_{3}$ 量子点仅在（200）晶面形成衍射峰, 其余锡掺杂 $\mathrm{CsPbB}_{3}$ 量子点均在 (100)、(110) 和 (200) 晶面出现了明显衍射峰, 与 PDF\#180364 号卡片对应的 $\mathrm{CsPbBr}_{3}$ 钙钛矿晶格参数相吻合 ${ }^{[14]}$ 。说明锡掺杂没有改变 $\mathrm{CsPbBr}_{3}$ 量子点的晶体结构。值得注意的是, 当 $\mathrm{SnBr}_{2}$ 添加量为 $0.05 \mathrm{mmol}$ 时, 所合成的锡掺杂 $\mathrm{CsPbr}_{3}$ 量子点各晶面衍射峰对应的强度都最强, 但 $\mathrm{SnBr}_{2}$ 添加 量为 $0.06 \mathrm{mmol}$ 时, 各衍射峰强度都最弱, 说明适量的锡掺杂可提高量子点的结 晶性能, 过量的锡掺杂则会对量子点的结晶产生负面影响。主要是由于 $\mathrm{Sn}^{2+}(1.12$ $\AA$ ) 离子半径小于 $\mathrm{Pb}^{2+}$ 离子半径 (1.19 $\AA$ ) [23], 当小粒径的 $\mathrm{Sn}^{2+}$ 离子替换更多的 $\mathrm{Pb}^{2+}$ 离子位置时, 使得锡掺杂 $\mathrm{CsPbBr}_{3}$ 钙钛矿晶体的容忍因子变小, 钙钛矿晶体 结构的对称性受到破坏，导致其结构稳定性降低 ${ }^{[24]}$ 。 


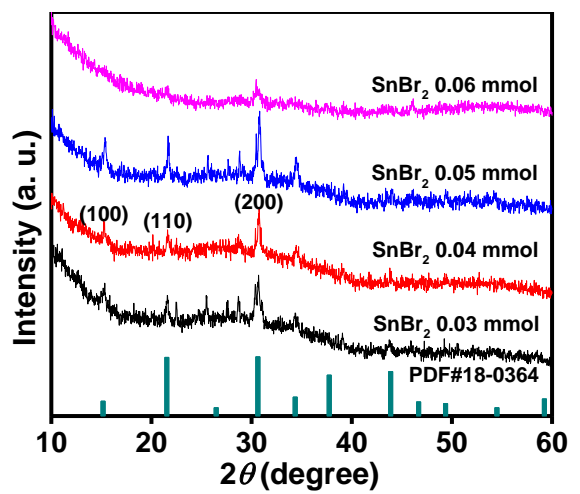

图 1 锡掺杂 $\mathrm{CsPbBr}_{3}$ 量子点的 XRD 图谱

Fig. 1. The XRD patterns of Tin doped $\mathrm{CsPBBr}_{3}$ quantum dots.

图 2 为锡掺杂 $\mathrm{CsPbBr}_{3}$ 量子点的 TEM 图谱。2 (a) - (c) 依次对应添加 $\mathrm{SnBr}_{2}$ 为 $0.03 \mathrm{mmol} 、 0.05 \mathrm{mmol}$ 和 $0.06 \mathrm{mmol}$ 所合成的锡掺杂 CSPbBr 3 量子点的 TEM 图谱。平均粒径分别为: $3.33 \mathrm{~nm}(0.03 \mathrm{mmol}), 2.91 \mathrm{~nm}(0.05 \mathrm{mmol})$ 和 $2.23 \mathrm{~nm}$ (0.06 mmol)。随着 $\mathrm{SnBr}_{2}$ 添加量的增加, 超小量予点粒径略微减小, 这主要是 由于 $\mathrm{Sn}^{2+}$ 离子的半径 $(1.12 \AA)$ 小于 $\mathrm{Pb}^{2+}$ 离乐径 (1.19 $\AA$ )。从插图中的 HRTEM 图谱可知, 所合成超小晶粒锡掺杂量子点伨在 (200) 晶面具有晶格间距约为 0.28 $\mathrm{nm}$ 的清晰晶格条纹。证明所合成的锡掺杂 $\mathrm{CsPbBr}_{3}$ 量子点具有相同于 $\mathrm{CsPbBr}_{3}$ 的晶体结构。图 3 是添加 $\mathrm{SnBr}_{2}$ 为 $0.05 \mathrm{mmol}$ 时所合成量子点的 $\mathrm{Cs} 、 \mathrm{~Pb} 、 \mathrm{Br}$ 和 $\mathrm{Sn}$ 元素的元素映射图像。结果显示, $\mathrm{Cs} 、 \mathrm{~Pb} 、 \mathrm{Br}$ 和 $\mathrm{Sn}$ 元素均匀分布于锡掺杂 $\mathrm{CsPbBr}_{3}$ 量子点表面。进一步对添加 $\mathrm{SnBr}_{2}$ 为 $0.05 \mathrm{mmol}$ 时所合成量子点的电子 能谱图 (EDS) 进行了分析, 如图 4 所示, 添加 $\mathrm{SnBr}_{2}$ 为 $0.05 \mathrm{mmol}$ 时所合成量 子点的 $\mathrm{Cs}$ Pb、 $\mathrm{Br}$ 和 $\mathrm{Sn}$ 元素的原子比分别为 $20.31 \% 、 8.68 \% 、 56.38 \%$ 和 4.63\%, 质量比分别为 $23.22 \% 、 33.30 \% 、 38.75 \%$ 和 $4.73 \%, \mathrm{Cs}:(\mathrm{Pb}+\mathrm{Sn}): \mathrm{Br}$ 原子比约为 1: 1: 3, 与 $\mathrm{CsPbBr}_{3}$ 晶体结构中的 Cs: $\mathrm{Pb}: \mathrm{Br}$ 原子比 1: 1: 3 相吻合 ${ }^{[25]}$ 。结合 图 3, 4 可以说明, 添加 $\mathrm{SnBr}_{2}$ 可实现锡离子部分替代 $\mathrm{CsPbBr}_{3}$ 量子点中的铅离 子, 合成锡掺杂 $\mathrm{CsPbBr}_{3}$ 量子点。 

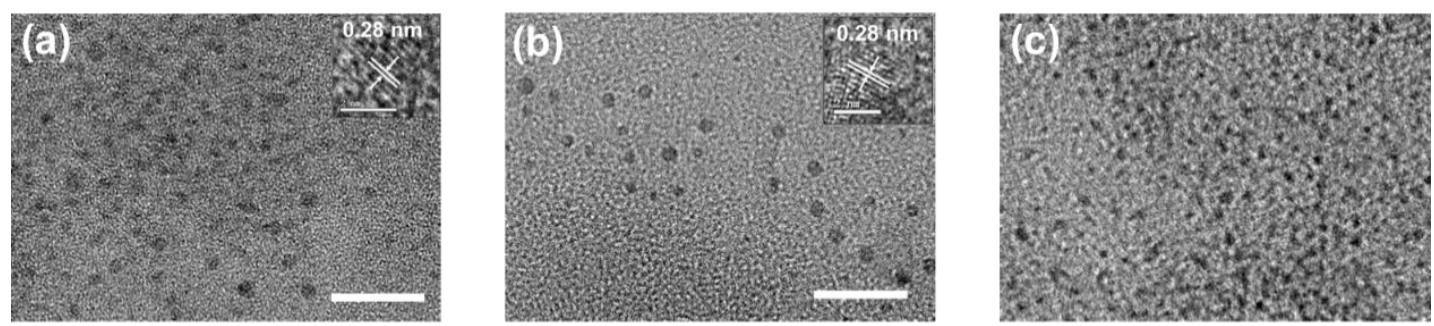

图 2 锡掺杂 $\mathrm{CsPbBr}_{3}$ 量子点的 TEM 图谱(标尺为 $20 \mathrm{~nm}$ )。（a） $\mathrm{SnBr}_{2} 0.03 \mathrm{mmol}$ ，(b) $\mathrm{SnBr}_{2}$

$0.05 \mathrm{mmol}$, (c) $\mathrm{SnBr}_{2} 0.06 \mathrm{mmol}$ 。插图为对应 TEM 图量子点的高分辨透射电镜（Highresolution transmission electron microscopy, HRTEM）图（标尺为 $2 \mathrm{~nm}$ ）。

Fig. 1. The TEM images of Tin doped $\mathrm{CsPbBr}_{3}$ quantum dots (scale bars represent $20 \mathrm{~nm}$ ). (a)

$\mathrm{SnBr}_{2} 0.03$ mmol. (b) $\mathrm{SnBr}_{2} 0.05 \mathrm{mmol}$. (c) $\mathrm{SnBr}_{2} 0.06 \mathrm{mmol}$. Inset pictures show the Highresolution transmission electron microscopy (HRTEM) of corresponding/quantum dots (scale bars represent $2 \mathrm{~nm})$.
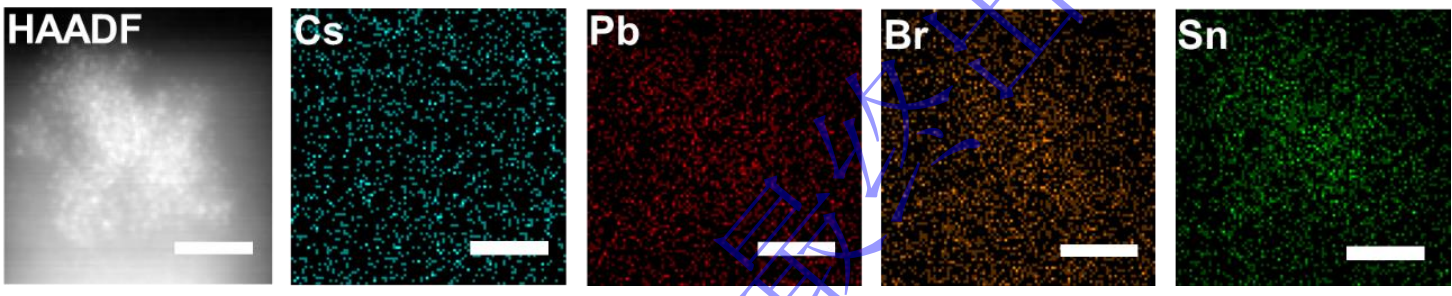

图 3 锡掺杂 $\mathrm{CsPbBr}$ 量子点的 $\mathrm{Cs} 、 \mathrm{~Pb} 、 \mathrm{Br}$ 和 $\mathrm{Sn}$ 元素的元素映射图像 $\left(\mathrm{SnBr}_{2} 0.05\right.$ $\mathrm{mmol}$ ), 标尺为 $50 \mathrm{~nm}$

Fig. 3. $\mathrm{Cs}, \mathrm{Pb}, \mathrm{Br}$, and $\mathrm{Sn}$ element mapping images of Tin doped $\mathrm{CsPbBr}_{3}$ quantum dots. $\left(\mathrm{SnBr}_{2}\right.$

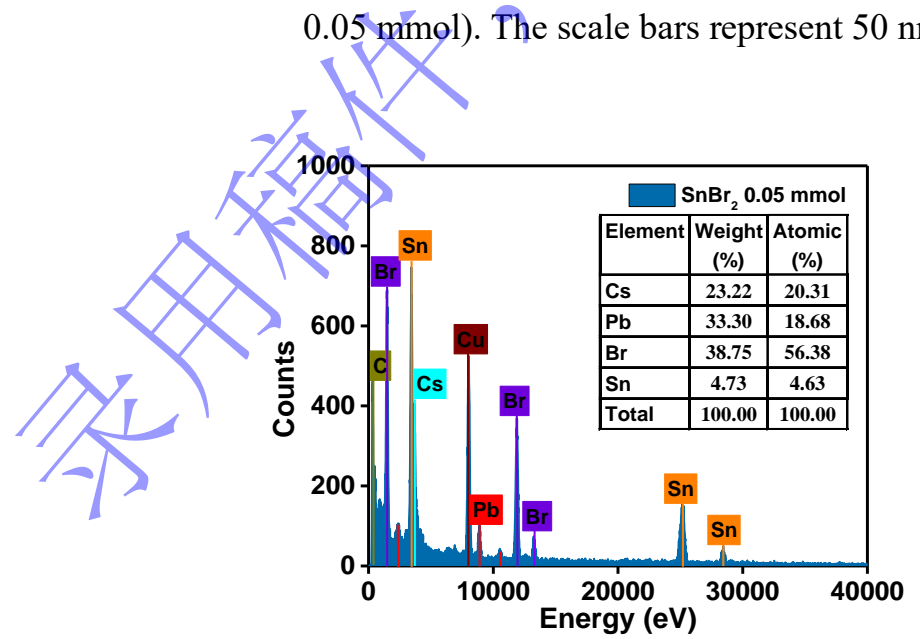

图 4 锡掺杂 $\mathrm{CsPbBr}_{3}$ 量子点的电子能谱图（ $\left.\mathrm{SnBr}_{2} 0.05 \mathrm{mmol}\right)$

Fig. 4. The Energy Dispersive System (EDS) of Tin doped $\mathrm{CsPbBr}_{3}$ quantum dots $\left(\mathrm{SnBr}_{2} 0.05\right.$ mmol). 


\section{2 光学特性}

\section{2 .1 吸收与苂光性能}

图 5 为 $\mathrm{Sn}^{2+}$ 离子掺杂量子点的吸收光谱和苂光光谱图。从图 5（a）可以看 出, 锡掺杂后量子点的吸收峰均在 $475 \mathrm{~nm}$ 附近。随着 $\mathrm{SnBr}_{2}$ 添加量的增加, 量 子点苂光发生蓝移 (激发波长为 $365 \mathrm{~nm}$ ), $\mathrm{SnBr}_{2}$ 添加量为 $0.03 \mathrm{mmol} 、 0.04 \mathrm{mmol}$ 、 $0.05 \mathrm{mmol}$ 和 $0.06 \mathrm{mmol}$ 时分别对应 $490 \mathrm{~nm} 、 482 \mathrm{~nm} 、 472 \mathrm{~nm}$ 和 $472 \mathrm{~nm}$ 的荧光 发射峰。其中, 添加 $\mathrm{SnBr}_{2}$ 为 $0.05 \mathrm{mmol}$ 时所合成量子点在 $472 \mathrm{~nm}$ 处的发射峰 最强, 属于蓝光发射。进一步对合成超小粒径量子点的 PLQY 采用积分球进行测 量, 对应的测试仪器为爱丁堡 FS 5 苂光光谱仪, 激发波长为 $365 \mathrm{~nm}$, 通过光学 积分球可以测量单位时间内激发光子数、被吸收局剩余的激发光子数、以及苂光 光子数, PLQY 可直接由苂光光子数除以吸收的光子数即可计算得出。结果如图 5 (c) 所示, 添加不同量 $\mathrm{SnBr}_{2}$ 所合成 $\mathrm{Sn}^{2+}$ 离子掺杂 $\mathrm{CsPbBr}_{3}$ 量子点的 PLQY 分 别为 $43.4 \%(0.03 \mathrm{mmol}) 、 32.3 \%(0.04 \mathrm{mmol}) 、 53.4 \%(0.05 \mathrm{mmol})$ 和 $21.7 \%(0.06$ $\mathrm{mmol}$ )。同样添加 $\mathrm{SnBr}_{2}$ 为 $0.05 \mathrm{mmol}$ 时合成的蓝光量子点 PLQY 最高。说明添 加 $\mathrm{SnBr}_{2}$ 为 $0.05 \mathrm{mmol}$ 时合成的蓝光量子点的光学性能最优异。当添加 $\mathrm{SnBr}_{2}$ 的 量为 $0.06 \mathrm{mmol}$ 时, 量子点苂发射峰虽仍位于 $472 \mathrm{~nm}$, 但与添加 $\mathrm{SnBr}_{2} 0.05 \mathrm{mmol}$ 时相同, 其 PLQY 迅速下降, 降至了 $21.7 \%$, 比添加 $\mathrm{SnBr}_{2} 0.05 \mathrm{mmol}$ 时降低了 $31.7 \%$ 。证明添加适量的 $\mathrm{SnBr}_{2}$ 合成的超小晶粒量子点具有更好的结晶性和更优 异的蓝光发射, 若 $\mathrm{SnBr}_{2}$ 添加过量, 则会导致超小晶粒量子点结晶性变差, 致使 其蓝光发射变弱。 

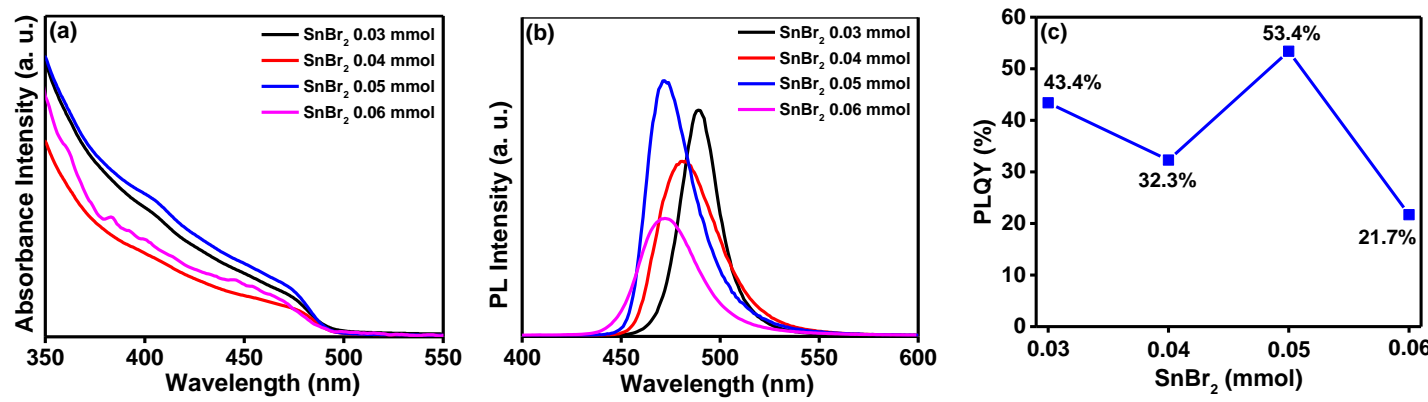

图 5 锡掺杂 $\mathrm{CsPbBr}_{3}$ 量子点的（a）吸收光谱，（b）苂光光谱，(c）苂光量子产率

(PLQY)

Fig.5. (a) Absorption spectra, (b) Emission spectra, and (c) PLQY of Tin doped C $\mathrm{SPBBr}$ quantum dots.

图 6 为锡掺杂 $\mathrm{CsPbBr}_{3}$ 量子点的苂光衰减曲线图。所有锡掺杂量子点均可采

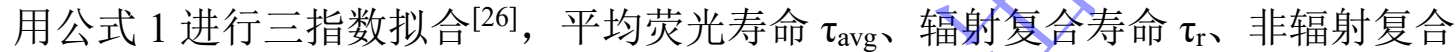
$\tau_{\mathrm{nr}}$ 、辐射复合衰减率 $\kappa_{\mathrm{r}}$ 和非辐射复合衰减率 $\kappa_{\mathrm{nr}} /$ 分别可采用公式 $2,3,4,5$ 和 6 进行计算 ${ }^{[27]}$ 。

$$
\begin{aligned}
& f(t)=A_{1} \exp \left(-\frac{t}{\tau_{1}}\right)+A_{2} \exp \left(-\frac{t}{\tau_{2}}\right)+A_{3} \exp \left(-\frac{t}{\tau_{3}}\right) \\
& \tau_{\text {avg }}=\sum\left(A_{i} \tau_{i}^{2}\right) / \sum A_{i} \tau_{i} \\
& \tau_{r}=\frac{\tau_{a v g}}{P L Q Y} \\
& \tau_{n r}=\frac{\tau}{1-P L Q V} \text { /avg } \\
& \kappa_{r}=\frac{1}{\tau_{r}}
\end{aligned}
$$

式中 $A_{1} 、 A_{2}$ 和 $A_{3}$ 为常数, $\tau_{1} 、 \tau_{2}$ 和 $\tau_{3}$ 分别短寿命、中等寿命和长寿命的衰减时 间。拟合结果如表 1 所示。从表 1 可以得出, 添加不同量 $\mathrm{SnBr}_{2}$ 合成的锡掺杂 $\mathrm{CsPbBr}_{3}$ 量子点的平均寿命 $\tau_{\mathrm{avg}}$ 分别为 $16.9 \mathrm{~ns}(0.03 \mathrm{mmol}) 、 15.81 \mathrm{~ns}(0.04 \mathrm{mmol})$ 、 $17.73 \mathrm{~ns}(0.05 \mathrm{mmol})$ 和 $14.78 \mathrm{~ns}(0.06 \mathrm{mmol})$ 。添加 $\mathrm{SnBr}_{2}$ 为 $0.05 \mathrm{mmol}$ 时所合 
成量子点对应的苂光寿命最长。为了研究锡掺杂后量子点的发光机制, 进一步对 辐射复合寿命 $\tau_{\mathrm{r}}$ 、非辐射复合寿命 $\tau_{\mathrm{nr}}$ 、辐射复合率 $\kappa_{\mathrm{r}}$ 和非辐射复合率 $\kappa_{\mathrm{nn}}$ 进行了 计算, 结果如表 2 所示。结果表明, 添加 $\mathrm{SnBr}_{2}$ 为 $0.05 \mathrm{mmol}$ 时所合成量子点对 应的辐射复合率 $\kappa_{\mathrm{r}}$ 最高, 非辐射复合率 $\kappa_{\mathrm{nr}}$ 最低, 说明添加 $\mathrm{SnBr}_{2}$ 为 $0.05 \mathrm{mmol}$ 时, 可有效降低超小晶粒蓝光锡掺杂量子点的非辐射复合缺陷密度。证明添加适 量的 $\mathrm{SnBr}_{2}$ 可有效降低超小晶粒锡掺杂 $\mathrm{CsPbBr}_{3}$ 量子点的非辐射复合率，提高超 小晶粒蓝光量子点的 PLQY ${ }^{[27,28]}$ 。

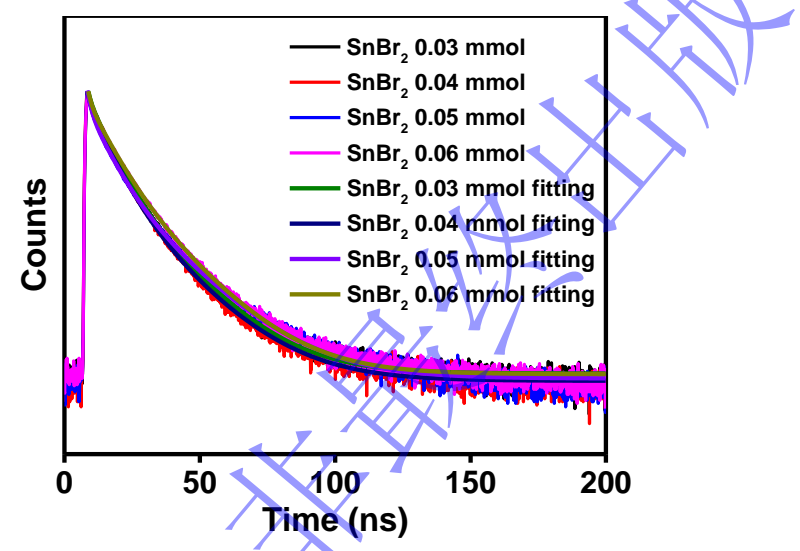

图 6 锡离子掺杂 $\mathrm{CsPbBr}_{3}$ 量子点的苂光衰减图

Fig. 6. The time-resolved $\mathrm{PL}$ decays of Tin doped $\mathrm{CsPbBr}_{3}$ quantum dots.

表而锡掺杂 $\mathrm{CsPbBr}_{3}$ 量子点的衰减曲线拟合参数

Table 1. The fitting results fitted by time-resolved PL decays curve of Tin doped $\mathrm{CsPBr}_{3}$ quantum

\begin{tabular}{|c|c|c|c|c|c|c|c|}
\hline $\begin{array}{c}\mathrm{SnBr}_{2} \\
\text { (mmol) } \\
\end{array}$ & $\begin{array}{l}A_{1} \\
(\%) \\
\end{array}$ & $\begin{array}{c}\tau_{1} \\
(\mathrm{~ns}) \\
\end{array}$ & $\begin{array}{c}\mathbf{A}_{2} \\
(\%) \\
\end{array}$ & $\begin{array}{c}\tau_{2} \\
(\mathrm{~ns}) \\
\end{array}$ & $\begin{array}{c}\mathbf{A}_{3} \\
(\%) \\
\end{array}$ & $\begin{array}{c}\tau_{3} \\
(\mathrm{~ns}) \\
\end{array}$ & $\begin{array}{c}\tau_{\text {avg }} \\
(\mathbf{n s}) \\
\end{array}$ \\
\hline 0.03 & 12.36 & 3.60 & 69.05 & 9.33 & 18.59 & 26.17 & 16.09 \\
\hline 0.04 & 4.52 & 1.83 & 69.01 & 9.16 & 26.48 & 22.92 & 15.81 \\
\hline 0.05 & 20.32 & 5.86 & 65.26 & 18.25 & 14.42 & 52.28 & 29.96 \\
\hline 0.06 & 4.42 & 1.65 & 69.56 & 8.10 & 26.03 & 21.64 & 14.78 \\
\hline
\end{tabular}


表 2 辐射复合寿命 $\tau_{\mathrm{r}}$ 、非辐射复合寿命 $\tau_{\mathrm{nr}}$ 、辐射复合衰减率 $\kappa_{\mathrm{r}}$ 和非辐射复合衰减率 $\kappa_{\mathrm{nr}}$ 计 算结果

Table 2. The calculate results of Radiative lifetime $\tau_{\mathrm{r}}$, Nonradiative lifetime $\tau_{\mathrm{nn}}$, Radiative decay rate $\kappa_{\mathrm{r}}$, and nonradiative decay rate $\kappa_{\mathrm{nr}}$

\begin{tabular}{ccccccc}
\hline $\begin{array}{c}\mathbf{S n B r}_{2} \\
(\mathbf{m m o l})\end{array}$ & $\begin{array}{c}\boldsymbol{\tau}_{\text {avg }} \\
(\mathbf{n s})\end{array}$ & $\begin{array}{c}\mathbf{P L Q Y} \\
(\boldsymbol{\%})\end{array}$ & $\begin{array}{c}\boldsymbol{\tau}_{\mathbf{r}} \\
(\mathbf{n s})\end{array}$ & $\begin{array}{c}\boldsymbol{\tau}_{\mathbf{n r}} \\
(\mathbf{n s})\end{array}$ & $\begin{array}{c}\boldsymbol{\kappa}_{\mathbf{r}} \\
\left(\times \mathbf{1 0}^{\mathbf{7}} \mathbf{s}^{-\mathbf{1}}\right)\end{array}$ & $\begin{array}{c}\boldsymbol{\kappa}_{\mathbf{n r}} \\
\left(\times \mathbf{1 0}^{7} \mathbf{s}^{-\mathbf{1}}\right)\end{array}$ \\
\hline 0.03 & 16.09 & 43.4 & 37.07 & 28.43 & 2.70 & 3.52 \\
0.04 & 15.81 & 32.3 & 48.95 & 23.35 & 2.04 & 4.28 \\
0.05 & 17.73 & 53.4 & 33.20 & 38.05 & 3.01 & 2.63 \\
0.06 & 14.78 & 21.7 & 68.11 & 18.88 & 1.47 & 5.30 \\
\hline
\end{tabular}

\section{3.2 发光稳定性}

最后, 为了以后更好地应用该蓝光量子点于光电器件领域, 对添加 $\mathrm{SnBr}_{2}$ 为 $0.05 \mathrm{mmol}$ 所合成的超小晶粒量子点的发光稳定性进行了研究。将其存放于大气 氛围 15 天 (重庆，湿度 $>60 \%$ ), 分别对存放第 $1 y / 2$-3、5、7、10 和 15 天的苂 光光谱进行了测试 (激发波长 $365 \mathrm{~nm}$ ), 前对存放第 $1 、 10$ 和 15 天后量子点的 PLQY 进行了测试, 结果如图 7 所少。量子点在空气中存放 15 天后, 其苂光发 射峰几乎没有明显改变, 存放第 $1 、 10$ 和 15 天蓝光量子点的 PLQY 依次为 $53.4 \%$ 、 $48.6 \%$ 和 $42.7 \%$, 即存放 15 矢后, $\mathrm{Sn}^{2+}$ 离子掺杂 $\mathrm{CsPbBr}_{3}$ 量子点仍具有 $472 \mathrm{~nm}$ 的 蓝光发射, 与存放第1 天的超小晶粒量子点的 PLQY 相比, 仅降低了 $10.7 \%$, 仍 保持第 1 天测试PLQY 的 $80 \%$ 。说明添加 $\mathrm{SnBr}_{2}$ 为 $0.05 \mathrm{mmol}$ 时合成的超小晶粒 锡掺杂 $\mathrm{CsPbB} 3$ 量子点在空气中具有良好的发光稳定性, 可应用于蓝光光电器件 领域。

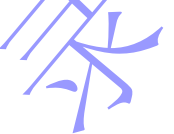




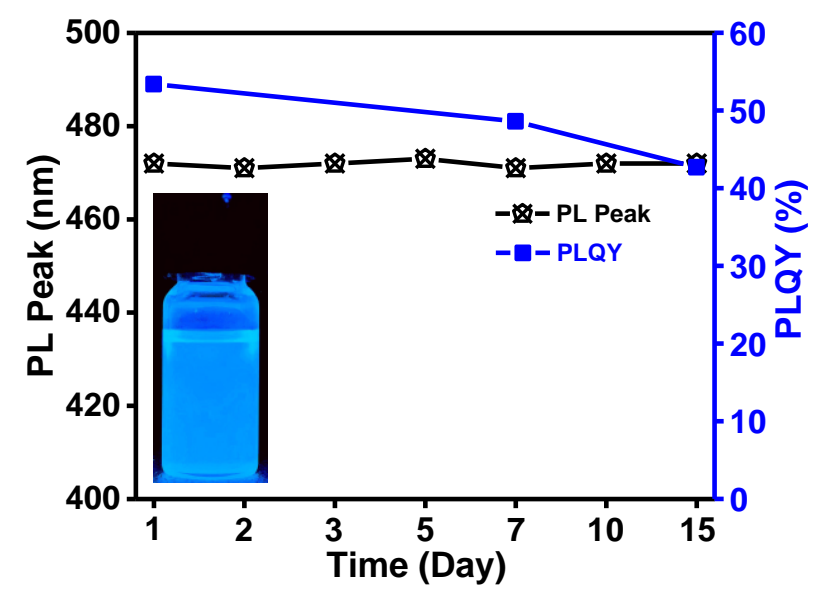

图 7 锡离子掺杂 $\mathrm{CsPBr}_{3}$ 量子点大气氛围存放 1-15 天的苂光峰位置及 $\mathrm{PLQY}$ 变化图

Fig. 7. The PL peak and PLQY of Tin doped $\mathrm{CsPbr}_{3}$ quantum dots from 1 to 15 days.

\section{4 结 论}

本文采用过饱和结晶的方法在室温下合成了粒径低手 $4 \mathrm{~nm}$ 的超小晶粒锡 ( $\mathrm{Sn}$ ) 掺杂 $\mathrm{CsPbBr}_{3}$ 量子点。通过调节添加 $\mathrm{SnBr}_{3}$ 量的不同, 合成了不同锡掺杂 $\mathrm{CsPbBr}_{3}$ 量子点, 对其结构特性和光学特性进行了研究。研究表明, 添加不同量 $\mathrm{SnBr}_{2}$ 合成的超小晶粒量子点的粒径随 $\mathrm{SnBr}_{2}$ 添加量的增加, 略微减小, 但晶格 间距几乎不变, 均为 $0.28 \mathrm{~nm}$ 。光吸收位置基本一致, 苂光发射光谱则随 $\mathrm{SnBr}_{2}$ 添 加量的增加出现了蓝移 $\mathrm{SnBr}_{2}$ 的添加量为 $0.05 \mathrm{mmol}$ 时所合成的约 $2.91 \mathrm{~nm}$ 的超小晶粒 $\mathrm{CsPbBr}$ 量余点 XRD 各晶面显示出最强衍射峰, 蓝光 (472 nm) PLQY 最高, 达到 $53.4 \%$, 空气中存放 15 天后, 苂光峰不发生明显变化, 仍是蓝光发 射, 其 PLQY 为 $42.7 \%$, 为合成量子点第一天测试的 $80 \%$, 证明该量子点具有良 好的发光稳定性。当 $\mathrm{SnBr}_{2}$ 添加量为 $0.06 \mathrm{mmol}$ 时, 合成的量子点仍为蓝光发射, 但其 PLQY 仅为 $21.7 \%$, 比添加 $\mathrm{SnBr}_{2}$ 为 $0.05 \mathrm{mmol}$ 时降低了 $31.4 \%, \mathrm{XRD}$ 各晶 面衍射峰变弱。证明添加适量的 $\mathrm{SnBr}_{2}$ 对 $\mathrm{CsPbBr}_{3}$ 量子点进行锡掺杂, 可有效提 高量子点的结晶性能和发光性能, 过量的添加 $\mathrm{SnBr}_{2}$ 则会对量子点的结晶产生负 面影响, 致使其发光性能降低。该方法合成的添加 $\mathrm{SnBr}_{2}$ 为 $0.05 \mathrm{mmol}$ 的超小晶 
粒、发光稳定以及 PLQY 高的蓝光量子点, 为合成苂光量子效率高的蓝光量子点 提供了参考。

参考文献

[1] Li C L, Han C, Zhang Y B, Zang Z G, Wang M, Tang X S, Du J 2017 Sol. Energy Mater. Sol. Cells 172341

[2] Li C L, Zang Z G, Han C, Hu Z P, Tang X S, Du J, Leng Y X, Sun K 2017 Nan̆o Energy 40195 [3] Song J Z, Tao F, Li J H, Xu L M, Zhang F J, Han B N, Shan Q S, Zeng HB 2018 Adv. Mater. 30 1805409

[4] Tang X S, Hu Z P, Chen W W, Xing X, Zang Z G, Hu W, Qiu J, Du J, Leng Y X, Jiang X F, Mai L Q 2016 Nano Energy 28462

[5] Zhang X, Lin H, Huang H, Reckmeier C, Zhang Y, Choy W C, Rogach A L 2016 Nano Lett. 16 1415

[6] Qu Z H, Chu Z M, Zhang X W, You J B 2019 Acta Phys. Sin. 68158504 (in Chinese) [翟子 涵, 储泽马, 张兴旺, 游经碧 2019 物理学报 68 158504]

[7] Shirasaki Y, Supran G J, Bawendi M G, Bulović V 2012 Nature Photon. 713

[8] Protesescu L, Yakunin S, Bodnarchuk M I, Krieg F, Caputo R, Hendon C H, Yang R X, Walsh A, Kovalenko M V 2015 Nano Let. 153692

[9] Liu F, Zhang Y H, Ding C. Kobayashi S, Izuishi T, Nakazawa N, Toyoda T, Ohta T, Hayase S, Minemoto T, Yoshino K, Dai S, Shen Q 2017 ACS Nano 1110373

[10] Duan C C, Cheng D, Yin Y, Chu L 2019 Acta Phys. Sin. 68158503 (in Chinese) [段聪聪, 程露, 殷禾, 珠琳 2019 物理学报 68 158503]

[11] Shi W-Q, Tian H, Lu Y X, Zhu H, Li F, Wang X X, Liu Y W 2021 Acta Phys. Sin. 70087303 (in Chinese) [石文奇, 田宏, 陆玉新, 朱虹, 李芬, 王小霞, 刘燕文 2021 物理学报 70 087303] [12] Chen W W, Xin X, Zang Z G, Tang X S, Li C L, Hu W, Zhou M, Du J 2017 J. Solid State Chem. 255115

[13] Guner T, Demir M M 2018 Phys. Status Solidi A 2151800120

[14] Li X M, Wu Y, Zhang S L, Cai B, Gu Y, Song J Z, Zeng H B 2016 Adv. Funct. Mater. 262435 [15] Bi C H, Wang S X, Li Q, Kershaw S V, Tian J J, Rogach A L 2019 J. Phys. Chem. Lett. 10943 
[16] Liu H W, Wu Z N, Shao J R, Yao D, Gao H, Liu Y, Yu W L, Zhang H, Yang B 2017 ACS Nano 112239

[17] van der Stam W, Geuchies J J, Altantzis T, van den Bos K H, Meeldijk J D, Van Aert S, Bals S, Vanmaekelbergh D, de Mello Donega C 2017 J. Am. Chem. Soc. 1394087

[18] Liu M, Zhong G H, Yin Y M, Miao J S, Li K, Wang C Q, Xu X R, Shen C, Meng H 2017 Adv. Sci. 41700335

[19] Li M, Zhang X, Matras-Postolek K, Chen H S, Yang P 2018 J. Mater. Chem. C 65506

[20] Pradeep K R, Chakraborty S, Viswanatha R 2019 Mater. Res. Express 6114004

[21] Wang H C, Wang W G, Tang A C, Tsai H Y, Bao Z, Ihara T, Yarita N/Tahara H, Kanemitsu Y, Chen S M, Liu R S 2017 Angew. Chem.Int. Edit. 5613650

[22] Zhang X T, Wang H, Hu Y, Pei Y X, Wang S X, Shi Z F, Colvin V L, Wang S N, Zhang Y, Yu W W 2019 J. Phys. Chem. Lett. 101750

[23] Zhang X L, Cao W Y, Wang W G, Xu B, Liu S, Dái HT, Chen S M, Wang K, Sun X W 2016 Nano Energy 30511

[24] Veldhuis S A, Boix P P, Yantara N, Li M, Sum T C, Mathews N, Mhaisalkar S G 2016 Adv. Mater. 286804

[25] Wang H C, Bao Z, Tsai H Y, Tang A C, Lu R S 2018 Small 141702433

[26] Huang H, Susha A S, Kershaw S V, Hung T F, Rogach A L 2015 Adv. Sci. 21500194

[27] Pan G C, Bai X, Xu W, Chen X, Zhai Y, Zhu J Y, Shao H, Ding N, Xu L, Dong B, Mao Y L, Song H W 2020 ACS Appl-Mater. Interfaces 1214195

[28] Wang S X, Wang Y, Zhang Y, Zhang X T, Shen X Y, Zhuang X W, Lu P, Yu W W, Kershaw S V, Rogach A L 2019 J Phys. Chem. Lett. 1090

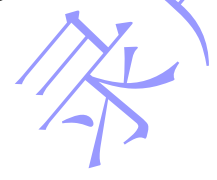


Synthesis and optical properties of ultra-small Tin doped $\mathrm{CsPbBr}_{3}$ blue Iuminescence quantum dots Zeng Fan-Ju $\left.{ }^{1) 2}\right)^{\dagger}$ Tan Yong-Qian ${ }^{1)}$ Wei Hu$^{2)^{\dagger}}$ Tang Xiao-Sheng ${ }^{2) 3)^{\dagger}}$ Zhang Xiao-Mei ${ }^{1)}$ Yin HaiFeng $^{1)}$

1) (School of Big Data Engineering, Kaili University, Kaili, Guizhou 556011, China)

2) (College of Optoelectronic Engineering, Chongqing University, Chongqing 400044, China)

3) (College of Optoelectronic Engineering, Chongqing University of Posts and Telecommunications, Chongqing 400065, China)

Abstract

All-inorganic perovskite $\mathrm{CsPbX}_{3}(\mathrm{X}=\mathrm{Cl} / \mathrm{Br}$ and I) quantum dots (QDs) have been wildly utilized in optoetectronic devices due to its tunable photoluminescence, high photoluminescence quantum yield (PLQY), and narrow-line width photoluminescence. However, the blue luminescence PLQY of $\mathrm{Cs} \mathrm{PbX}_{3}$ perovskite quantum dots is still lag behind their redand green luminescence (PLQYs nearly 100\%) counterparts. Herein, we present a facile strategy to synthetic ultrasmall blue luminescence Tin doped $\mathrm{CsPbBr}_{3}$ perovskite QDs by supersaturated recrystallization synthetic approach at room temperature, and the particle size of as-prepared QDs is lower than 4 $\mathrm{nm}$. The crystal structure and optical property of Tin doped $\mathrm{CsPbr}_{3}$ QDs are characterized by XRD, TEM, ultraviolet-visible spectrophotometer, and fluorescence spectrophotometer. The results 
show that the particle size of as-prepared QDs is slightly shrink from $3.33 \mathrm{~nm}\left(\mathrm{SnBr}_{2} 0.03 \mathrm{mmol}\right)$ to $2.23 \mathrm{~nm}\left(\mathrm{SnBr}_{2} 0.06 \mathrm{mmol}\right)$ as the $\mathrm{SnBr}_{2}$ adding amount increase, but there is no obvious change in the lattice spacing of doped QDs. The partial $\mathrm{Pb}$ for Tin replacement leads to a blue-shift from $490 \mathrm{~nm}\left(\mathrm{SnBr}_{2} 0.03 \mathrm{mmol}\right)$ to $472 \mathrm{~nm}\left(\mathrm{SnBr}_{2} 0.06\right.$ mmol) of the optical spectra. The highest PLQY and the strongest XRD diffraction of ultra-small Tin doped $\mathrm{CsPBr}_{3}$ blue luminescence QDs is obtained by adding $\mathrm{SnBr}_{2} 0.05 \mathrm{mmol}$, and the blue luminescence located at $472 \mathrm{~nm}$ with the PLQY of $53.4 \%$. There is no any change in PL peak of Tin doped $\mathrm{CsPbBr}_{3}$ QDs $\left(\mathrm{SnBr}_{2} 0.05 \mathrm{mmol}\right)$ by storing it under the ambient atmosphere for 15 days and the PLQY of $\mathrm{Sn}^{2+}$ doped QDs is still remain $80 \%$ of the initial after 15 days. It is concluded that the crystallization and optical property could be effectively improved

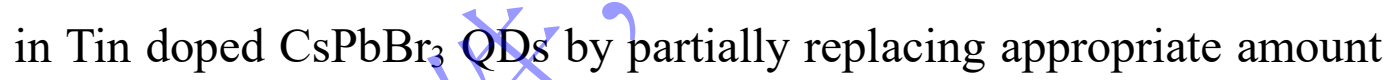
of $\mathrm{Pb}$ by Tin.

Keywords: Ultra-small, Tin doped $\mathrm{CsPbBr}_{3}$ quantum dots, Synthesis, Blue luminescence, 1

Optical property, Stable luminescence

* Project supported by Science and Technology Foundation of Guizhou Province (grant No. ZK $[2021] 245)$, the Project supported by the National Natural Science Foundation of China (Grant No. 61975023，61875211, 51602033，61520106012), the Doctoral Project of Kaili University (Grant Nos. BS202004, BS201301), the Academic New Seedling Cultivation and Innovation Exploration Special Project of Kaili University (Grant No. Qian Ke He Ping Tai Ren Cai [2019]01-4), and the Major Research 
Projects of Innovative Groups in Education Department of Guizhou Province of China (Grant No. Qian Jiao He KY[2018]035).
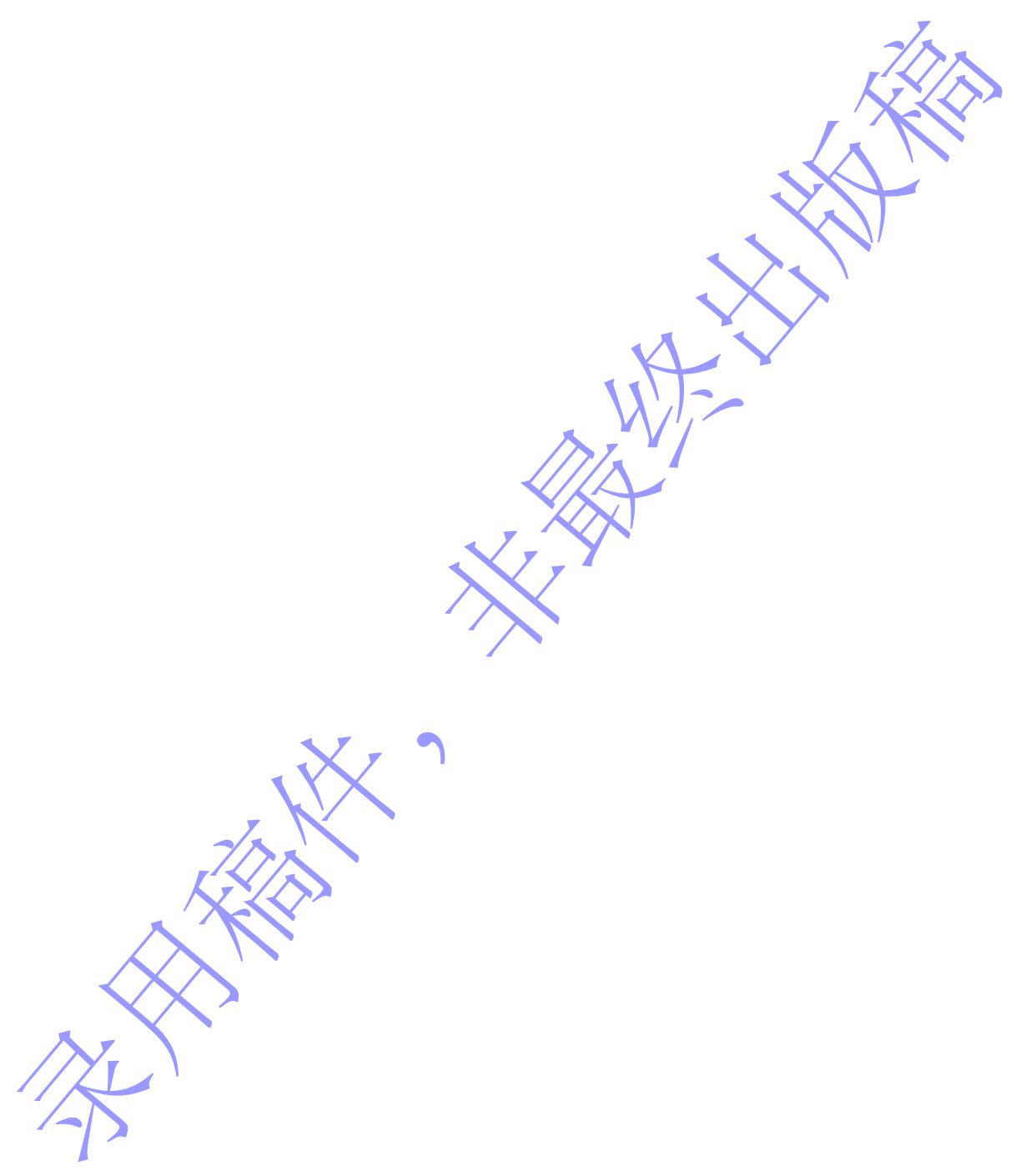